\title{
Comunicação científica no Brasil (1998-2012): indexação, crescimento, fluxo e dispersão'
}

\author{
Scientific communication in Brazil (1998-2012): \\ Indexing, growth, flow and dispersion
}

\author{
Rogério MUGNAINI² \\ Luciano Antonio DIGIAMPIETRI ${ }^{3}$ \\ Jesús Pascual MENA-CHALCO ${ }^{4}$
}

\section{Resumo}

A produção científica brasileira vem demonstrando ascensão no cenário mundial, o que pode decorrer da internacionalização da ciência nacional e/ou das revistas nacionais. Nesse contexto, este estudo reuniu a produção científica brasileira das bases Web of Science e Scientific Electronic Library Online entre 1998 e 2012, com o fim de analisar como a internacionalização tem influenciado a performance do país. Com base em Zonas de Bradford da produção das diversas áreas, buscou avaliar o aumento do número de revistas em cada uma das zonas, assim como a proporção de artigos publicados em revistas nacionais, ao longo de cinco triênios. Observou-se que o número de revistas indexadas exclusivamente na Scientific Electronic Library Online cresceu no período, com exceção do triênio 4, quando aproximadamente 60\% da produção nacional foi publicada em revistas estrangeiras (Web of Science). Notou-se que o aumento mais generalizado do número de revistas das áreas deu-se primeiramente na zona 3 (triênio 2004-2006), depois na zona 2 (triênio 2007-2009) e finalmente na zona 1 (triênio 2010-2012). Já o percentual da produção em revistas nacionais diferenciou três grupos de áreas: ciências sociais e humanas, onde as zonas 1 e 2 são exclusivas de revistas nacionais, e a zona 3 vem dando lugar a publicações estrangeiras; Física, Ciência do Espaço e Imunologia, cuja produção é exclusivamente internacional; e as demais áreas, de ciências exatas e da terra, saúde e biológicas, que vêm aumentando o percentual nas zonas 1 e 2, permanecendo a zona 3 direcionada a publicações internacionais.

Palavras-chave: Avaliação de pesquisa. Brasil. Produção científica. Revistas científicas nacionais. Zonas de Bradford.

\begin{abstract}
The Brazilian scientific production growth in the international scenario may be due to the internationalization of Brazilian science and journals. Within this context, this study collected Brazilian scientific production from the Web of Science and Scientific Electronic Library Online databases - data from 1998 to 2012 - in order to analyze how internationalization influenced the performance of the country. Based on the Bradford Zones and considering the different fields, the aim of this study is to assess the growth in the number of journals in each zone, as well as the proportion of the papers published in national journals throughout five triennia. It was possible to observe that the number ofjournals exclusively indexed in Scientific Electronic Library Online increased in the period, except in the fourth triennium when several journals were also indexed in the Web of Science. However, about $60 \%$ of the national production is published in international journals (Web of Science). The most generalized growth in the number of journals from different fields occurred initially in zone 3 (triennium

\footnotetext{
1 Trabalho apresentado no VII Seminário Internacional sobre Estudos Quantitativos e Qualitativos da Ciência e Tecnologia"Prof. Gilberto Sotolongo Aguilar" no XIII Congresso Internacional de Informação - INFO'2014. Habana, Cuba.

2 Universidade de São Paulo, Escola Comunicações e Artes, Departamento de Biblioteconomia e Documentação. Av. Prof. Lúcio M. Rodrigues, 443, 05508020, São Paulo, SP, Brasil. Correspondência para/Correspondence to: R. MUGNAINI.E-mail:<mugnaini@usp.br>.

3 Universidade de São Paulo, Escola de Artes, Ciências e Humanidades. São Paulo, SP, Brasil.

4 Universidade Federal do ABC, Centro de Matemática, Computação e Cognição. Santo André, SP, Brasil.

Recebido em 3/6/2014, reapresentado em 11/8/2014 e aceito para publicação em 1/9/2014.
} 
2004-2006), then in zone 2 (triennium 2007-2009), and finally in zone 1 (triennium 2010-2012). On the other hand, the percentage of production in national journals across the fields presented three different behaviors: in Social and Human Sciences zones 1 and 2 contains exclusively national journals, and zone 3 is receiving international journals; in Physics, Space Science and Immunology production is exclusively international; in other fields such as Exact \& Earth, Health and Biological Sciences there is an increasing percentage of production in national journals in zone 1 and 2, but zone 3 remains international.

Keywords: Research evaluation. Brazil. Scientific production. National scientific journals. Bradford zones.

\section{Introdução}

O levantamento da produção científica de um país permite estudar um dos aspectos que podem ser qualificados como resultados mensuráveis do amplo sistema de ciência, tecnologia e inovação. Acompanhar o fluxo de comunicação científica das diversas áreas facilita o processo de avaliação da pesquisa, cujas características são tão diversificadas quanto o é a própria ciência.

A análise quantitativa da ciência, que se dá a partir de indicadores, é tida como complementar à análise qualitativa, realizada pelos pares em diversas etapas, desde a formação, com a entrada e progressão na carreira de cientista, até a avaliação das pesquisas empreendidas, consubstanciada em manuscritos e títulos.

Nesse sentido, diferentes abordagens foram consideradas para avaliar e analisar o impacto da indexação de revistas científicas em contextos regionais ou locais. Testa (2011) destaca a expansão da cobertura de revistas nacionais na Web of Science (WoS) de diversas regiões. O fenômeno foi analisado em diversos trabalhos e contextos, como, por exemplo: Quevedo-Blasco (2013) apresenta uma visão geral das revistas ibero-americanas de Psicologia indexadas no Journal Citations Reports (JCR) de 2011; Purnel e Quevedo-Blasco (2013) analisam o impacto das revistas espanholas na WoS, identificando após 2005 um expressivo incremento, tanto da produção científica quanto da indexação espanhola; Collazo-Reyes (2013) apresenta e discute o aumento do número de revistas indexadas na WoS, no conjunto de revistas da América Latina e do Caribe, devido principalmente à política de abertura dos critérios de seleção da WoS.

Similarmente, nesses últimos anos, estudos bibliométricos foram realizados para analisar a produção científica indexada na WoS de diferentes áreas, em diversos países: Canadá (Wolfram, 2012), Colômbia (Rojas-Sola \& de San-Antonio-Gomez, 2010), Croácia (Pikic et al., 2012), Coreia (Huh, 2013; Fink et al., 2014), Malásia (Abrizah et al., 2013) e Portugal (Costa et al., 2013).
No Brasil, a análise de indicadores da ciência remonta aos anos 70 (Morel, R. \& Morel, C., 1977), quando o país apresentava uma produção na casa das centenas de artigos indexados nas bases do Institute for Scientific Information (ISI), atual Thomson Reuters, e ocupava posição próxima à $30^{a}$ no ranking mundial.

Leta (2011) apresenta um conjunto de 16 estudos que têm como foco indicadores de resultado da ciência brasileira, publicados em revistas, e cujo período de análise cobre pelo menos 5 anos. Um deles é o já mencionado estudo de Morel e Morel, enquanto três outros trabalhos cobrem a década de 1990, e outros doze abrangem o período de 2000 em diante, o que aponta a atualidade desses estudos. A autora afirma ainda que as agências de fomento, como o Ministério de Ciência e Tecnologia (MCT) e a Fundação de Amparo à Pesquisa do Estado de São Paulo (FAPESP) têm se encarregado do esforço de divulgação de estatísticas e indicadores de ciência e tecnologia, após a virada do milênio - porém, iniciativas de análise do fenômeno, como visto, são esporádicas.

Durante a última década pôde-se notar maior envolvimento da comunidade científica nacional com o tema dos indicadores bibliométricos, o que decorreu de implementações dos critérios de avaliação do amplo sistema orquestrado pela Coordenação de Aperfeiçoamento de Pessoal de Nível Superior (CAPES). Criado em 1976 para avaliação de desempenho de programas de pós-graduação (Guimarães \& Humann, 1995), vem tendo seus critérios aprimorados gradativamente, passando a apresentar uma abordagem mais objetiva a partir de 1998 (Souza \& Paula, 2002).

Analisando os critérios de avaliação das diversas áreas no triênio 2007-2009, Miranda e Mugnaini (2013) observaram que a produção intelectual é um dos cinco itens que compõem o formulário. Algumas áreas atribuem pontuação de 40\%, e outras 35\% - os demais itens medidos são a proposta do programa, o quadro docente, 
as dissertações e teses de alunos e a inclusão social. Já o peso da publicação em revistas científicas varia de 30\% a $65 \%$, peso esse que, multiplicado pelo peso da produção intelectual, resultará no peso da publicação em periódicos na avaliação total do programa, que varia de 12\% (áreas de Artes e Música) a 26\% (Ciência da Computação e Matemática, Probabilidade e Estatística). Segundo os autores, um critério predominante tem sido o fator de impacto do JCR, que crescentemente vem servindo como o principal critério de classificação das revistas consideradas mais importantes, seguido da indexação das revistas em índices de citação como WoS, Scopus e Scientific Electronic Library Online (SciELO) (Mugnaini \& Sales, 2011).

Essa conjuntura que se delineou no Brasil expressa a relação direta entre indicadores bibliométricos e política científica, tornando os estudos quantitativos da ciência uma utilidade nacional, porém mantendo excessiva dependência de produtos e sistemas de indicadores bibliométricos oferecidos por empresas como Thomson e Elsevier (Mugnaini, 2013).

Em contrapartida, as revistas nacionais, que desde o início da década de 90 recebiam financiamento das agências de fomento, visando estimular a disseminação do conhecimento gerado por teses, assim como a produção científica de áreas de interesse local (Guimarães \& Humann, 1995), têm logrado significativo reconhecimento nacional e internacional, sinalizando uma mudança de papel no fluxo de comunicação científica nacional. Nesse sentido, desde 1998, o Projeto SciELO, atuante junto aos editores nacionais, tem conferido não apenas maior visibilidade à pesquisa nacional (para a própria nação), mas dinamizado a editoria de revistas científicas (Packer \& Meneghini, 2007). Também, a partir de 2005, as revistas vêm demonstrando indexação progressiva na Web of Science (Testa, 2011), proporcionando o aumento da produção brasileira naquela base (Leta, 2011). Além disso, desde o início de 2014, passam a compor o SciELO Citation Index as citações recebidas de revistas indexadas na Web of Science (Packer, 2014), agregadas às citações nacionais (já computadas pelo Projeto SciELO desde o ano 2000), passando a unir as bases regionais à denominada Principal Coleção da Web of Science (Thomson Reuters, 2014).

O processo de seleção de revistas do SciELO se baseia em critérios de mérito científico rigorosos, entre outros aspectos, resultando que houve casos de revistas que conseguiram indexação antes na Web of Science. Mugnaini e Sales (2011) observaram que a validade de tais critérios tem sido amplamente reconhecida pela comunidade científica nacional, podendo ser notada pelo fato de 37\% (17) das áreas de avaliação haverem considerado a indexação no SciELO como um critério para classificação de revistas de mais alto estrato (A1).

Packer (2011) compara a posição dos vinte países mais bem posicionados no ranking mundial, tanto em número de artigos quanto em número de revistas indexados na Web of Science, no ano de 2010. O autor apresenta interessantes constatações a respeito da diferença de posição que um mesmo país ocupa em cada ranking. Como exemplo destacam-se Holanda e Suíça, cuja posição no ranking de revistas é cerca de dez posições à frente do ranking de artigos, devido à concentração de títulos de editoras comerciais desses países. 0 autor apresenta como caso inverso a situação da Coreia do Sul, Índia e China, com posição de destaque no ranking de artigos, o que, segundo ele, pode decorrer de inconsistência na qualidade da editoração científica nacional ou na aplicação dos critérios exigidos para indexação. Sobre o Brasil, cuja posição no ranking de artigos (13ª) está pouco melhor que no ranking de revistas (15), Packer conjectura ser devido à subindexação das revistas nacionais.

Por outro lado, Leta (2012) esclarece que a maior presença dos autores brasileiros na Web of Science - em grande parte, fruto da presença de novos títulos nacionais na base - ocasionou a diminuição do percentual de artigos brasileiros em inglês, de 93,6\% (2001) para 82,4\% (2010). Diante dessa constatação, alerta para a necessidade de iniciativas complementares, como a própria internacionalização das revistas - que poderiam passar a publicar artigos na língua franca - e a proficiência de pesquisadores, para que a ciência ganhe audiência internacional.

Considerando o contexto delineado neste estudo, de acordo com os tópicos relacionados que compuseram o escopo temático das cinco últimas conferências da (Internacional Society for Scientometrics and Informetrics, 2014, online), observa-se que tanto "avaliação da política científica" quanto "indicadores de ciência e tecnologia" são tópicos gerais presentes em cada edição: Suécia (2005), Espanha (2007), Brasil (2009), África do Sul (2011) e Áustria (2013). Já os "estudos de produtividade em 
nível nacional" deixaram de figurar na última edição do evento; por outro lado, é na edição brasileira que o tópico sobre "comparação entre bases de dados e cobertura" surge, perdurando até 2013; ademais, apenas a edição brasileira considerou o tópico "revistas não-anglófonas", denotando dedicação exclusiva ao tema.

Tais constatações permitem afirmar que estudos de produção científica nacional, com vistas à geração de indicadores para subsídio à Política Científica, são alvo de interesse mundial, tendo a discussão sobre cobertura de bases de dados uma crescente atenção, (o que pode se dever à recente alternativa oferecida pela base Scopus, em relação à hegemonia da Web of Science). Contudo o tema relacionado às revistas não-anglófonas (e sua internacionalização) encontra menor interlocução, denotando assim a necessidade de ser discutido em âmbito nacional.

Diante do exposto, este estudo pretende analisar a produção científica nacional, a partir da agregação da produção científica brasileira registrada nas bases WoS e SciELO, considerando a influência de três fenômenos concomitantes observados por Mugnaini et al. (2014): (1) o crescimento do número de publicações em revistas internacionais, como resultado da política científica vigente há mais de uma década; (2) a crescente indexação de revistas brasileiras na WoS, na última década; e (3) o aumento da coleção de revistas exclusivamente indexadas na base SciELO.

Além de uma análise de produtividade, este estudo investiga o aumento, fluxo e dispersão da produção científica nacional. Para tanto, define as Zonas de Bradford das diversas áreas temáticas, buscando avaliar o aumento do número de revistas em cada uma das zonas, assim como a proporção de artigos publicados em revistas nacionais, ao longo de cinco triênios de produção. A abordagem busca oferecer uma caracterização da importância das revistas de diferentes zonas, na medida em que concentram maior ou menor percentual de artigos de determinada área, oferecendo assim novos parâmetros para a avaliação da produção científica nacional.

\section{Métodos}

O período de análise foi delimitado a cinco triênios completos de avaliação de programas de Pós-
-Graduação brasileira (1998 e 2012). O início do período coincide tanto com a criação da SciELO quanto com o momento em que a Capes passa a dar à avaliação um caráter mais objetivo, enquanto o fim do período coincide com o final do último triênio. Esse período de tempo é abrangente o suficiente para que possam ser observados possíveis efeitos, decorrentes da adoção de critérios de avaliação específicos, no perfil da produção científica nacional, a cada triênio, sendo esse o motivo das análises haverem sido realizadas trienalmente.

As fontes de dados foram as bases WoS e SciELO, consistindo dos registros bibliográficos de artigos (originais ou de revisão) que apresentassem pelo menos um autor com endereço institucional do Brasil, considerando variações que atendessem a diversos idiomas (BRAZIL, BRASIL ou BRESIL).

Da WoS foi recuperado um total de 402845 registros, dos quais selecionaram-se somente os artigos originais ou de revisão publicados em revistas, restando 310195 deles, uma vez descartadas as publicações em anais de conferências. A recuperação foi realizada automaticamente em setembro de 2013, com o auxílio de um macro de programação desenvolvido por Milanez (2011). Já os registros bibliográficos dos artigos originais ou de revisão da SciELO foram entregues pelos mantenedores da base, mediante solicitação, em janeiro de 2014, somando 162 582. Descartadas as sobreposições entre as bases, chegou-se a um total de 396650 registros.

As revistas exclusivas de cada base foram identificadas como tal ("SciELO" ou "WoS"), e revistas indexadas em ambas receberam identificação "SciELO/WoS", sendo os dados destas últimas obtidos da base que apresentasse o maior número de artigos. Essa diferença pode advir do fato de a revista haver sido indexada em uma base alguns anos antes da indexação na outra.

Foi considerado como ano de indexação das revistas SciELO aquele que a própria SciELO divulga como ano de entrada. Tal procedimento fez com que revistas que tiveram fascículos anteriores inseridos (indexação retrospectiva) fossem consideradas no início do período da análise como não indexadas (em 1998 somaram 1500 artigos, chegando a 2180 em 2001, e decrescendo até o final do período, chegando a zero em 2012). Por outro lado, revistas indexadas na SciELO em 2013, cujos artigos de 2012 foram indexados retrospectivamente, não 
compuseram o corpus da pesquisa - a não ser no caso de a publicação ter sido indexada até 2012 na WoS. No caso da WoS, por falta de acesso à informação sobre o ano exato de indexação, foi considerado o ano mais antigo, segundo os artigos presentes na base.

A organização da produção segundo áreas do conhecimento consistiu na adoção da classificação do Essential Science Indicators ${ }^{\circledR}$ (ESI), que agrupa aproximadamente 250 áreas de classificação de revistas da WoS (ambas da Thomson Reuters) em 22 áreas - às quais foi adicionada a área de Ciências Humanas, originalmente não contemplada naquele produto. No caso das revistas indexadas na WoS, foi utilizada a lista de revistas (Journal List) que a própria instituição disponibiliza em livre acesso no Science Watch (Thomson Reuters, 2013). Já para as revistas da SciELO, utilizou-se a classificação segundo categorias da WoS, para posterior reclassificação segundo áreas do ESI, consultando as definições temáticas (Field Definitions) do Science Watch.

Para análise da comunicação científica de cada área, com vistas à determinação do conjunto de revistas importantes para as áreas, foi utilizada a Lei de Dispersão de Bradford (Bradford, 1961). O uso dessa metodologia permitiu identificar três conjuntos de revistas (ou Zonas de Bradford) de acordo com a quantidade de artigos publicados, sendo que cada zona concentra um terço da produção de uma área específica. A Zona 1 reúne um número reduzido de revistas, com grande quantidade de artigos cada uma; a Zona 2 apresenta um número maior de revistas, porém com menores quantidades de artigos cada uma; e a Zona 3 mostra maior dispersão, com muitas revistas, cada qual com poucos artigos. A primeira zona contém as revistas mais representativas do processo de comunicação científica das áreas; a segunda zona, as revistas em consolidação; e a terceira, as revistas novas, as que estão deixando de ser utilizadas e as que são utilizadas ocasionalmente. Cada revista é classificada em uma única área pela ESI. Definidas as Zonas de Bradford da produção científica das diferentes áreas em cada triênio, foi possível analisar as variações do número de revistas de cada zona, bem como da proporção de artigos em revistas nacionais.

A nacionalidade da revista utilizou a seguinte agregação, baseada na região de publicação declarada pelo editor: nacional (Brasil), regional (América Latina \&
Caribe, exceto Brasil) e internacional (demais países não considerados nas categorias anteriores).

\section{Resultados e Discussão}

O fluxo de comunicação científica no Brasil apresenta um cenário diversificado no período analisado, considerando a indexação das revistas brasileiras nas bases SciELO e WoS (Mugnaini et al., 2014). Tal quadro revela a entrada crescente de revistas indexadas exclusivamente na SciELO em todos os triênios, com destaque para o quarto triênio, quando um expressivo número de revistas brasileiras foi incluído na WoS, com a expansão realizada pela Thomson Reuters (Testa, 2011), entre 2006 e 2008.

Contudo, considerando-se o fluxo de artigos nessas revistas, nota-se que a maior parte da produção nacional se dá em revistas indexadas somente na WoS (Mugnaini et al., 2014): no primeiro triênio, período de criação da base SciELO, os artigos em revistas WoS representaram $73,1 \%$, percentual decrescente que atinge o valor mínimo de 54,7\% em 2007, e cresce ao longo do último triênio, atingindo 59,0\% em 2012.

Certamente a maior presença de revistas brasileiras implicou aumento do número de artigos, e consequentemente, maior representatividade da pesquisa nacional na WoS. Por outro lado, é necessário analisar o aumento da produção brasileira, separando o aumento do número de artigos em revistas nacionais, do aumento em revistas internacionais.

Na Tabela 1 observa-se que a taxa de crescimento percentual da produção em revistas estrangeiras indexadas na WoS apresenta crescimento estável entre os triênios 2 e 4, com desaceleração no último triênio (com mais de 66 mil artigos publicados), sendo a soma de crescimento no período de 124,3\%. A produção de revistas internacionais na base SciELO ou revistas SciELO/WoS - que apresentam significativo aumento diz respeito às coleções dos projetos SciELO de outros países, como África do Sul, Espanha, Portugal e algumas revistas do SciELO Saúde Pública.

É entre as revistas brasileiras (categoria nacional) indexadas na SciELO ocorre a maior taxa de crescimento. Aquelas indexadas também na WoS somam um cresci- 
mento de $442,6 \%$ no período, com destaque para os triênios 2 e 4, cujo aumento decorre diretamente da indexação das revistas na WoS, e apenas 16,2\% no último triênio (com mais que 29 mil artigos). Quanto às revistas indexadas somente na SciELO, a maior taxa de crescimento se dá do triênio 2 (quando da inclusão do primeiro conjunto de revistas da base), decrescendo até uma taxa negativa de -18,1\% no triênio 4 (quando diversas revistas entram também na WoS), voltando a crescer $(23,6 \%)$ no último triênio (com a publicação de mais de 19 mil artigos) e somando $345,9 \%$ de crescimento no período. A produção em revistas nacionais indexadas exclusivamente na WoS mostra aumentos altíssimos, claramente resultante da entrada das revistas naquela base nos triênios 3 e 4.

Já as revistas regionais, que correspondem àquelas publicadas em outros países latino-americanos, apresentam menor quantidade de artigos, também demonstrando aumentos altíssimos quando elas são indexadas também na WoS. Contudo, entre as revistas exclusivas da SciELO, nota-se um aumento constante, acumulando 402,9\% no período completo, denotando a importância das revistas da região para a ciência brasileira - ainda que tenham pequena expressão em números absolutos.

Dada a menor quantidade de revistas SciELO, o aumento da base ou indexação na WoS apresenta sensível taxa de crescimento. Esse fenômeno é acentuado pelo alto percentual de artigos de brasileiros nas revistas nacionais como um todo. Segundo Collazo-Reyes (2013), no período entre 2005 e 2011 as revistas SciELO/ WoS brasileiras apresentaram 88,8\% de endogenia - ou seja, apenas 10,2\% artigos de autores estrangeiros -, realidade que contrasta com países como Argentina,

Tabela 1. Distribuição e crescimento percentual trienal da produção científica brasileira, segundo a região de publicação da revista e a base de indexação - período de 1998-2012.

\begin{tabular}{|c|c|c|c|c|c|c|c|}
\hline \multirow{3}{*}{$\begin{array}{l}\text { Base } \\
\text { Triênio }\end{array}$} & \multicolumn{7}{|c|}{ Região de publicação da revista } \\
\hline & \multicolumn{2}{|c|}{ International } & \multicolumn{2}{|c|}{ Regional } & \multicolumn{2}{|c|}{ Nacional } & \multirow{2}{*}{$\begin{array}{c}\text { Total } \\
\text { Frequência }\end{array}$} \\
\hline & Frequência & Crescimento \% & Frequência & Crescimento $\%$ & Frequência & Crescimento \% & \\
\hline WoS & 212593 & 124,3 & 1135 & 1155,7 & 12665 & 1644,1 & 226393 \\
\hline 1 & 22522 & - & 160 & - & 3201 & - & 25883 \\
\hline 2 & 30171 & 34,0 & 67 & $-58,1$ & 45 & $-98,6$ & 30283 \\
\hline 3 & 40319 & 33,6 & 32 & $-52,2$ & 560 & 1144,4 & 40911 \\
\hline 4 & 53206 & 32,0 & 437 & 1265,6 & 3683 & 557,7 & 57326 \\
\hline 5 & 66375 & 24,8 & 439 & 0,5 & 5176 & 40,5 & 71990 \\
\hline SCIELO/WOS & 8873 & 175,1 & 1881 & 14027,1 & 70434 & $442,6 \%$ & 81188 \\
\hline 1 & 731 & - & 1 & - & 1986 & - & 2718 \\
\hline 2 & 1047 & 43,2 & 138 & 13700,0 & 5680 & 186,0 & 6865 \\
\hline 3 & 1807 & 72,6 & 191 & 38,4 & 8710 & 53,3 & 10708 \\
\hline 4 & 2255 & 24,8 & 703 & 268,1 & 25003 & 187,1 & 27961 \\
\hline 5 & 3033 & 34,5 & 848 & 20,6 & 29055 & 16,2 & 32936 \\
\hline SCIELO & 1478 & 310,9 & 2702 & 402,9 & 61316 & 345,9 & 65496 \\
\hline 1 & 81 & - & 85 & - & 2746 & - & 2912 \\
\hline 2 & 106 & 30,9 & 159 & 87,1 & 10289 & 274,7 & 10554 \\
\hline 3 & 180 & 69,8 & 377 & 137,1 & 17049 & 65,7 & 17606 \\
\hline 4 & 355 & 97,2 & 730 & 93,6 & 13969 & $-18,1$ & 15054 \\
\hline 5 & 756 & 113,0 & 1351 & 85,1 & 17263 & 23,6 & 19370 \\
\hline Não indexada & 3079 & & 659 & & 18835 & & 22573 \\
\hline Total & 226023 & 57,1 & 6377 & 1,6 & 163250 & 41,3 & 395650 \\
\hline
\end{tabular}

Fonte: elaborado pelos autores (2014).

Nota: Região da revista, baseada no país de publicação, declarado pelo editor: nacional (Brasil), regional (América Latina \& Caribe, exceto Brasil) ou internacional (demais países não considerados nas categorias anteriores); SciELO: Scientific Electronic Library Online; WOS: Web of Science. 
Chile e México, cujo percentual de endogenia é próximo de $67 \%$, segundo o autor.

Esses resultados reforçam a já citada necessidade de internacionalização das revistas científicas (Leta, 2011), uma vez que atualmente o ingresso na WoS não depende do idioma. Por outro lado, Collazo-Reyes (2013) destaca que a maior presença da pesquisa brasileira na WoS fez com que no período de 2005-2011 o português se tornasse o segundo idioma mais frequente (após o inglês) dos artigos publicados em revistas latino-americanas. 0 debate ora suscitado relembra os questionamentos da década de 1990, quando se discutia a exclusão de países não-anglófonos, porém agora exigindo o passo seguinte: internacionalização da ciência brasileira.

Nesse sentido, a análise da produção brasileira nas áreas do conhecimento pode ser mais detalhada, ao se comparar o percentual de artigos em revistas internacionais, assim como sua variação ao longo do tempo: observou-se pouca alteração, com crescimento médio de 57\%, e leve crescimento durante o triênio 5, que compreende o período de 2010 a 2012 (Mugnaini et al., 2014). Tal constatação sugere que a desaceleração do crescimento da base SciELO (Packer \& Meneghini, 2007), assim como o aumento da produção em revistas internacionais crescentemente estimulado pela avaliação nacional, está influenciando o crescimento relativo da produção em revistas internacionais.

Torna-se então necessário considerar que a produção científica distribui-se em conjuntos de revistas, que diferem quanto à magnitude do número de artigos, devendo-se ainda distinguir a produção nas diversas áreas.

A Figura 1 apresenta a comparação do tamanho das Zonas de Bradford total e em cada área. Em ordem decrescente do número de revistas que compõem a zona 1, observa-se primeiramente que as 113 revistas que a compõem são responsáveis por $1 / 3$ da produção nacional no período entre 1998 e 2012, enquanto as zonas 2 e 3 apresentam, respectivamente, 593 e 8383 revistas. As áreas de Medicina Clínica, Engenharia e Matemática são as que apresentam maior número de revistas na zona 1, com 26, 21 e 19 revistas respectivamente. Já nas áreas de Ciência do Espaço e Multidisciplinar, identifica-se apenas uma revista na zona 1 - revistas que publicaram no período completo um total de 898 e 744 artigos, respectivamente. Considerando a linha de tendência que sobrepõe as barras, pode-se observar a área Multidisciplinar é a menos expressiva, com um total de 1676 artigos, em contraste com Medicina Clínica (87 036), Ciência de Plantas e Animais (50 148), Ciências Sociais (31 332), Física (30 467), Química (29 514) e Ciências Agrárias (28 201).

Quanto à comparação entre o tamanho das zonas, por meio da Correlação de Spearman entre o percentual de revistas por zona em cada área, observa-se maior intensidade na correlação negativa entre as zonas 2 e 3 (-0,983), significando que as áreas em que a zona 3 é mais representativa são aquelas nas quais a representatividade da zona 2 é menor. Destacaram-se como áreas de maior dispersão, ou seja, com maior percentual de revistas na zona 3: Ciências Sociais (zona 2 com 2,6\% do total de revistas, e zona 3 com 96,8\%), Psiquiatria/Psicologia (zona 2 com 2,4\%, e zona 3 com 96,4\%) e Economia e Negócios (zona 2 com 3,0\%, e zona 3 com 95,6\%). Contrariamente, as áreas com maior zona 2 são: Imunologia (zona 2 com 14,3\%, e zona 3 com 80,2\%), Matemática (zona 2 com 14,6\%, e zona 3 com 81,0\%) e Ciência da Computação (zona 2 com 13,3\%, e zona 3 com 82,2\%).

Tendo conhecido a distribuição do número de revistas e de artigos segundo as bases de indexação e a magnitude das zonas das diversas áreas, é necessário analisar a presença das revistas nacionais na publicação da ciência brasileira.

Para tanto, considerou-se, na Figura 2, não a base de indexação, mas o país de publicação das revistas nacionais, avaliando-se o percentual dos artigos publicados nessas revistas, nas zonas de cada área. Uma primeira observação é que a zona 1 apresenta o maior percentual de artigos em revistas nacionais na maioria das áreas. A zona 2, por sua vez, apresenta maior percentual que a zona 3, com exceção apenas da área de Microbiologia. Esse comportamento decrescente é bem demarcado quando se observam os percentuais das zonas correspondentes à produção nacional total (Figura 1), representada como Brasil, que anteriormente encontrava-se na primeira posição).

Ainda na Figura 2, pode-se notar um conjunto de cinco áreas cujas zonas 1 e 2 são predominantemente de revistas nacionais: Ciências Humanas, Economia e Negócios, Psiquiatria/Psicologia, Ciências Agrárias e Ciências Sociais. Considerando que a zona 3 apresenta 


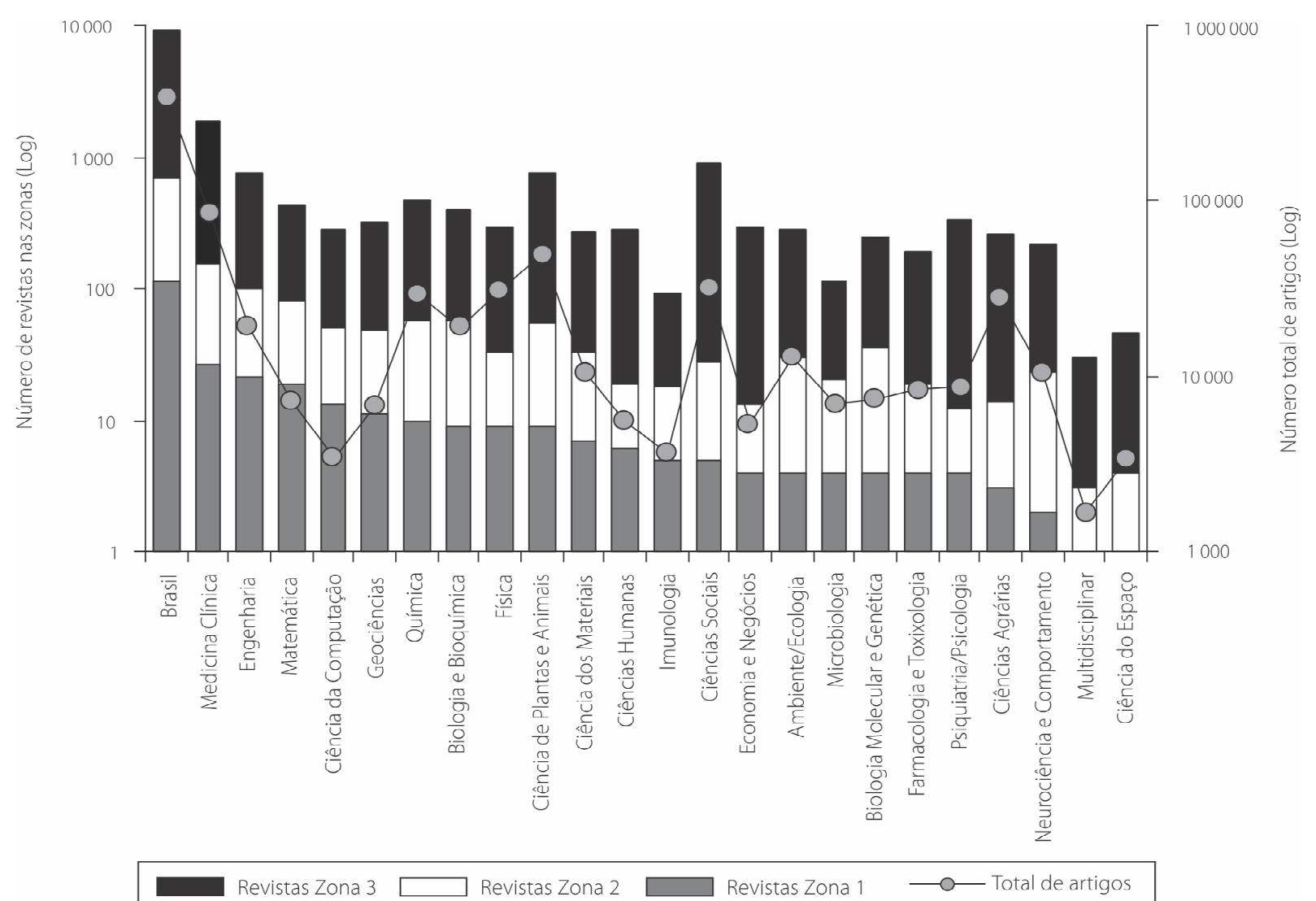

Figura 1. Produção científica brasileira, segundo o tamanho das Zonas de Bradford (número de revistas) e o número de artigos, por áreas temáticas - período de 1998-2012.

Fonte: Elaborado pelos autores (2014).

mais dispersão entre um conjunto maior de revistas, observa-se que, dessas áreas, apenas Ciências Humanas apresenta mais alto percentual $(57,9 \%)$ de artigos em revistas nacionais, sendo a área de menor internacionalização na sua produção. A área de Ciências Sociais apresenta comportamento similar, diferindo apenas no fato de que, na zona 2, a produção em revistas nacionais representa 90,2\%, e na zona 3, 53,6\%. Entre as demais áreas pode-se observar que a zona 3 é predominantemente composta de artigos em revistas internacionais, concentrando a maioria das áreas de saúde e biológicas mais ao centro do gráfico, com a zona 1 apresentando percentual de artigos em revistas nacionais variando de 71,7\% (Medicina Clínica) a 94,1\% (Biologia e Bioquímica). Já no extremo direito predominam as áreas de exatas e da Terra, com zona 1 variando de 0\% (Física, Imunologia e Ciência do Espaço) a 68,8\% (Química). Finalmente, destaca-se o maior percentual de artigos em revistas nacionais na zona 2, nas áreas de Ciência de Plantas e Animais e Farmacologia e Toxicologia.

A ordenação das áreas segundo o percentual de artigos brasileiros na zona 1, observada na Figura 2, vai ao encontro das evidências apresentadas por Leite et al. (2011), que analisaram o percentual de pesquisadores com perfil de publicação predominantemente internacional, com base em seus currículos Lattes. Contudo, o presente estudo permite análises mais criteriosas das revistas que compõem as zonas 2 e 3 , podendo revelar peculiaridades do fluxo de comunicação científica das áreas, à medida que revistas internacionais começam a surgir e serem consolidadas em área de perfil predominantemente nacional e vice-versa.

Pelo fato de esta análise não pretender entrar no nível das revistas em si, mas da evolução das zonas, apresentam-se a seguir duas tabelas que, com base nos dados utilizados para a elaboração das duas figuras 


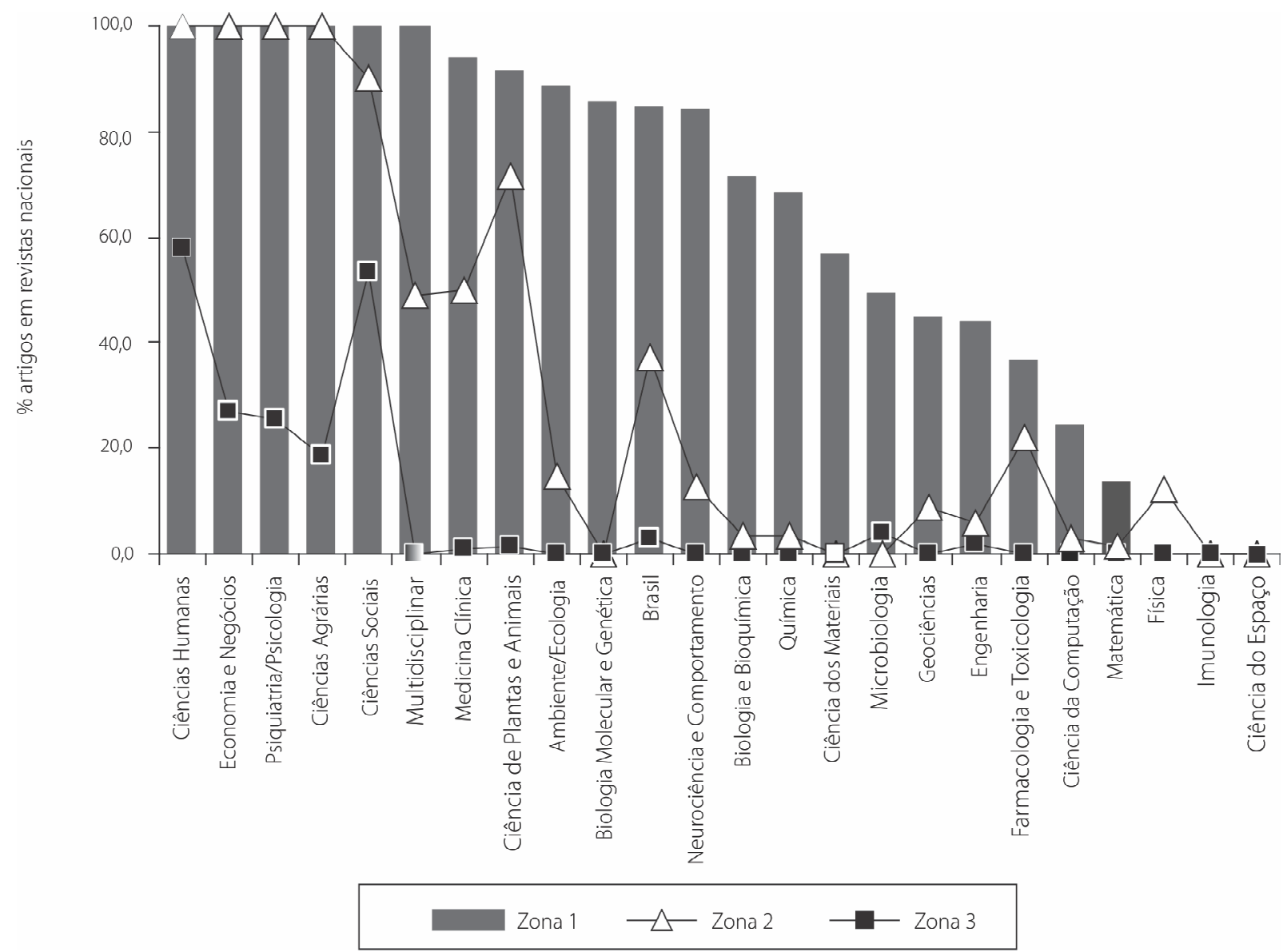

Figura 2. Porcentagem de artigos em revistas nacionais, por Zonas de Bradford e área - período de 1998-2012.

Fonte: Elaborado pelos autores (2014).

anteriores, permitem a análise da variação do tamanho das zonas de cada área, ao longo dos triênios.

Na Tabela 2 pode-se observar que, considerando o total da produção nacional (Brasil), há aumento no número de revistas das zonas, entre todos os triênios.

De maneira geral, como é de se esperar, a zona 3 é a que mais apresenta aumento (+) do número de revistas entre os triênios. Geralmente nessa zona agregam-se revistas novas, nas quais a comunidade de uma área passa a publicar. Com base nos números mínimo e máximo de revistas dos triênios, ao longo do período, observa-se que áreas como Ciências Humanas, Ciências Sociais e Multidisciplinar apresentam aumento destacado. Por outro lado, algumas áreas têm o número de revistas reduzido (-) entre alguns triênios, como exemplo a Física, cuja redução é observada entre os triênios 2-3 e 4-5.
À medida que se sobe para as zonas 2 e 1, respectivamente, observa-se menor número de aumentos (+) entre as áreas, bem como maior número de diminuições (-).

Os números mínimo e máximo das áreas na zona 2 permitem destacar as áreas com maior aumento, em ordem decrescente: Ciências Humanas, Ciências Agrárias, Economia e Negócios, Ciências Sociais e Psiquiatria/Psicologia. São exatamente as mesmas áreas que na Figura 2 apresentaram predominância de artigos em revistas nacionais nessa zona.

Observando a Tabela 3, a seguir, pode-se considerar que, destas áreas, Ciências Agrárias e Psiquiatria/ Psicologia apresentam redução desse percentual no último triênio, enquanto Ciências Sociais apresenta oscilação. 
A ordenação da Tabela 2 se baseia no número máximo de revistas por triênio, na zona 1. Observa-se que, na base, com menor número de revistas, encontra-se a maioria das áreas recém-mencionadas, mais a área de Ciência do Espaço e Multidisciplinar. Já as exatas e da terra estão no topo da Tabela, com exceção de Medicina Clínica e Ciência de Plantas e Animais - na verdade as áreas de saúde e biológicas encontram-se dispersas entre o conjunto.

Ainda sobre a Tabela 2, é importante mencionar que a maior ocorrência de aumento das áreas se dá entre os triênios 2-3 (na zona 3), 3-4 (na zona 2) e 4-5 (na zona
1). Pode-se associar esse fenômeno ao sistema de avaliação coordenado pela Capes desde 1998 (Souza \& Paula, 2002), uma vez que seu efeito mais imediato se dá entre as revistas de menor frequência (das zonas 3 das áreas) entre os triênios 2-3, ou seja, entre os anos 2003 e 2004. Consequentemente, chega às zonas 2 na transição das avaliações trienais entre os anos 2006 e 2007, e, finalmente, às zonas 1 entre os anos 2009 e 2010. Trata-se do Qualis, que consiste na classificação dos veículos (revistas, anais de conferência e livros) utilizados pelos programas de pós-graduação para publicação de suas pesquisas (Coordenação de Aperfeiçoamento de Pessoal

Tabela 2. Variação (positiva, negativa ou nula), mínimo e máximo do número de revistas das zonas das diversas áreas entre os triênios - período de 1998-2012.

\begin{tabular}{|c|c|c|c|c|c|c|c|c|c|c|c|c|c|c|c|c|c|c|}
\hline \multirow{3}{*}{ Áreas } & \multicolumn{18}{|c|}{ Número de revistas } \\
\hline & \multicolumn{6}{|c|}{ Zona1 } & \multicolumn{6}{|c|}{ Zona2 } & \multicolumn{6}{|c|}{ Zona3 } \\
\hline & $1-2$ & $2-3$ & $3-4$ & $4-5$ & mín & máx & $1-2$ & $2-3$ & $3-4$ & $4-5$ & mín & máx & $1-2$ & $2-3$ & $3-4$ & $4-5$ & mín & máx \\
\hline Brasil & + & & + & + & 56 & 122 & + & + & + & + & 357 & 564 & + & + & + & + & 3.375 & 6.233 \\
\hline Medicina Clínica & + & + & + & + & 9 & 30 & - & + & + & - & 66 & 109 & + & + & + & + & 662 & 1.357 \\
\hline Engenharia & - & $=$ & + & + & 14 & 19 & + & + & + & + & 58 & 69 & + & + & + & + & 284 & 471 \\
\hline Matemática & + & + & $=$ & $=$ & 13 & 17 & + & + & + & - & 42 & 57 & + & + & + & + & 148 & 274 \\
\hline Ciência da Computação & + & $=$ & + & + & 3 & 14 & + & $=$ & + & + & 21 & 36 & + & + & + & + & 72 & 159 \\
\hline Química & + & - & - & + & 8 & 11 & + & - & + & - & 35 & 42 & + & + & + & + & 221 & 301 \\
\hline Ciência de Plantas e Animais & + & + & + & + & 4 & 11 & - & - & + & + & 22 & 45 & + & + & + & + & 330 & 556 \\
\hline Geociências & + & - & $=$ & $=$ & 8 & 10 & + & - & + & - & 25 & 33 & + & + & + & + & 111 & 208 \\
\hline Biologia e Bioquímica & + & - & + & + & 5 & 10 & + & $=$ & + & + & 28 & 48 & + & + & + & + & 185 & 262 \\
\hline Física & - & $=$ & + & - & 7 & 8 & $=$ & - & + & + & 18 & 22 & + & - & + & - & 168 & 197 \\
\hline Ciência dos Materiais & - & $=$ & $=$ & + & 6 & 8 & - & $=$ & + & + & 19 & 23 & + & + & + & - & 109 & 168 \\
\hline Ciências Humanas & $=$ & + & + & + & 2 & 8 & + & + & + & + & 3 & 12 & - & + & + & + & 44 & 147 \\
\hline Imunologia & $=$ & $=$ & + & + & 4 & 7 & $=$ & + & + & + & 9 & 13 & + & + & - & + & 33 & 53 \\
\hline Ambiente/Ecologia & - & $=$ & $=$ & $=$ & 3 & 7 & $=$ & $=$ & + & + & 19 & 27 & + & + & + & + & 90 & 205 \\
\hline Ciências Sociais & $=$ & + & + & + & 3 & 7 & + & + & + & + & 9 & 23 & + & + & + & + & 173 & 548 \\
\hline Neurociência e Comportamento & $=$ & + & + & + & 1 & 6 & + & + & + & + & 11 & 23 & + & + & + & + & 104 & 154 \\
\hline Microbiologia & - & + & - & + & 3 & 5 & + & + & + & + & 10 & 17 & + & + & + & + & 46 & 79 \\
\hline Psiquiatria/Psicologia & $=$ & + & + & $=$ & 2 & 5 & + & + & + & + & 4 & 10 & + & + & + & + & 98 & 208 \\
\hline Biologia Molecular e Genética & + & - & - & + & 1 & 5 & + & - & & $=$ & 14 & 26 & + & + & + & + & 88 & 160 \\
\hline Ciências Agrárias & + & $=$ & + & + & 1 & 5 & $=$ & + & + & + & 4 & 12 & + & + & + & - & 101 & 183 \\
\hline Economia e Negócios & + & + & + & + & 1 & 5 & $=$ & + & + & + & 3 & 9 & + & + & + & + & 60 & 182 \\
\hline Farmacologia e Toxicologia & $=$ & $=$ & $=$ & + & 3 & 4 & + & + & + & + & 9 & 18 & + & + & + & + & 63 & 136 \\
\hline Ciência do Espaço & - & $=$ & $=$ & + & 1 & 2 & + & $=$ & + & - & 2 & 4 & $=$ & + & - & + & 19 & 32 \\
\hline Multidisciplinar & $=$ & $=$ & $=$ & $=$ & 1 & 1 & $=$ & $=$ & $=$ & $=$ & 2 & 2 & - & + & + & + & 6 & 23 \\
\hline
\end{tabular}

Fonte: Elaborado pelos autores (2014).

Nota: Foram utilizados sinais indicando se as mudanças entre triênios subsequentes foram de "+" (crescimento), "-" (decrescimento) ou "=" (inalterado). Valores mínimo (mín) e máximo (máx) correspondem aos valores extremos observados entre os triênios, não importando qual. 
de Nível Superior, 2014). Em relação às revistas, consiste em uma lista disponibilizada às comunidades científicas de quase 50 áreas de avaliação, com as seguintes classificações: A1 (mais alta) e A2, B1 a B5 (mais baixa) e $C$ (não científicas). A lista de cada área aumenta a cada trênio, à medida que a produção se dispersa por novas revistas, conforme observado na zona 3. Além disso, a classificação segundo estratos distingue revistas, ação que pode explicar o aumento do número de artigos em algumas delas, repercutindo assim nas zonas 1 e 2 .

Na Tabela 3, observando o total da produção nacional (Brasil), nota-se alta concentração de artigos em revistas nacionais na zona 1 (mínimo 79\% e máximo 86\%), valores que decrescem drasticamente para a zona 2 (mínimo 24\% e máximo 43\%) e se reduzem a 2\% (mínimo e máximo) para a zona 3.

Este é outro efeito relacionados com os critérios de avaliação, uma vez que o estímulo à publicação em revistas internacionais vem crescentemente se alastrando ao longo do período, e no quarto triênio (2007-2009) o Fator de Impacto passa a ser adotado por diversas áreas, como Ciências Sociais (Administração, Contabilidade \& Turismo), dobrando o valor mínimo do indicador de 0,5 para 1,0 no último triênio. Ressalta-se que, no presente

Tabela 3. Variação (positiva, negativa ou nula), mínimo e máximo do percentual de artigos em revistas nacionais nas zonas das diversas áreas, entre os triênios - período de 1998-2012.

\begin{tabular}{|c|c|c|c|c|c|c|c|c|c|c|c|c|c|c|c|c|c|c|}
\hline \multirow{3}{*}{ Áreas } & \multicolumn{18}{|c|}{ Percentual de artigos em revistas nacionais } \\
\hline & \multicolumn{6}{|c|}{ Zona1 } & \multicolumn{6}{|c|}{ Zona2 } & \multicolumn{6}{|c|}{ Zona3 } \\
\hline & $1-2$ & $2-3$ & $3-4$ & $4-5$ & mín & máx & $1-2$ & $2-3$ & $3-4$ & $4-5$ & mín & máx & $1-2$ & $2-3$ & $3-4$ & $4-5$ & mín & máx \\
\hline Ciências Agrárias & $=$ & $=$ & $=$ & $=$ & $100 \%$ & $100 \%$ & $=$ & $=$ & $=$ & - & $86 \%$ & $100 \%$ & - & - & + & - & $17 \%$ & $43 \%$ \\
\hline Ciências Humanas & $=$ & $=$ & $=$ & $=$ & $100 \%$ & $100 \%$ & $=$ & $=$ & $=$ & $=$ & $100 \%$ & $100 \%$ & + & - & + & + & $31 \%$ & $66 \%$ \\
\hline Economia e Negócios & $=$ & $=$ & $=$ & $=$ & $100 \%$ & $100 \%$ & + & $=$ & $=$ & $=$ & $91 \%$ & $100 \%$ & + & + & - & - & $0 \%$ & $45 \%$ \\
\hline Multidisciplinar & $=$ & $=$ & $=$ & $=$ & $100 \%$ & $100 \%$ & $=$ & $=$ & + & + & $0 \%$ & $69 \%$ & $=$ & $=$ & $=$ & $=$ & $0 \%$ & $0 \%$ \\
\hline Psiquiatria/Psicologia & $=$ & $=$ & $=$ & $=$ & $100 \%$ & $100 \%$ & $=$ & $=$ & $=$ & - & $95 \%$ & $100 \%$ & + & - & - & - & $16 \%$ & $43 \%$ \\
\hline Ciências Sociais & $=$ & $=$ & $=$ & - & $89 \%$ & $100 \%$ & + & - & + & & $88 \%$ & $93 \%$ & + & - & - & - & $49 \%$ & $63 \%$ \\
\hline Medicina Clínica & $=$ & $=$ & $=$ & - & $88 \%$ & $100 \%$ & + & - & - & + & $35 \%$ & $58 \%$ & - & + & + & - & $0 \%$ & $1 \%$ \\
\hline Ciência de Plantas e Animais & $=$ & $=$ & - & - & $88 \%$ & $100 \%$ & + & - & + & - & $36 \%$ & $83 \%$ & + & + & - & - & $1 \%$ & $3 \%$ \\
\hline Neurociência e Comportamento & $=$ & - & - & - & $65 \%$ & $100 \%$ & $=$ & + & + & - & $0 \%$ & $16 \%$ & $=$ & $=$ & $=$ & $=$ & $0 \%$ & $0 \%$ \\
\hline Biologia Molecular e Genética & - & + & + & - & $52 \%$ & $100 \%$ & $=$ & $=$ & $=$ & $=$ & $0 \%$ & $0 \%$ & $=$ & $=$ & $=$ & $=$ & $0 \%$ & $0 \%$ \\
\hline Biologia e Bioquímica & - & + & - & - & $63 \%$ & $89 \%$ & + & - & $=$ & $=$ & $0 \%$ & $7 \%$ & + & - & $=$ & $=$ & $0 \%$ & $1 \%$ \\
\hline Ambiente/Ecologia & + & - & + & $=$ & $29 \%$ & $100 \%$ & + & + & - & + & $0 \%$ & $28 \%$ & $=$ & $=$ & + & - & $0 \%$ & $2 \%$ \\
\hline Brasil & + & + & + & - & $79 \%$ & $86 \%$ & + & + & + & - & $24 \%$ & $43 \%$ & + & - & + & - & $2 \%$ & $2 \%$ \\
\hline Geociências & - & + & + & + & $17 \%$ & $76 \%$ & + & - & + & - & $0 \%$ & $6 \%$ & $=$ & $=$ & $=$ & $=$ & $0 \%$ & $0 \%$ \\
\hline Química & - & + & + & - & $64 \%$ & $74 \%$ & - & + & + & - & $0 \%$ & $8 \%$ & $=$ & $=$ & $=$ & $=$ & $0 \%$ & $0 \%$ \\
\hline Farmacologia e Toxicologia & + & + & - & + & $0 \%$ & $68 \%$ & $=$ & $=$ & + & - & $0 \%$ & $25 \%$ & - & $=$ & $=$ & $=$ & $0 \%$ & $5 \%$ \\
\hline Microbiologia & + & - & + & - & $42 \%$ & $66 \%$ & $=$ & $=$ & $=$ & $=$ & $0 \%$ & $0 \%$ & $=$ & $=$ & $=$ & $=$ & $0 \%$ & $0 \%$ \\
\hline Ciência dos Materiais & + & - & + & - & $40 \%$ & $60 \%$ & $=$ & + & - & $=$ & $0 \%$ & $7 \%$ & $=$ & $=$ & $=$ & $=$ & $0 \%$ & $0 \%$ \\
\hline Engenharia & + & - & - & + & $25 \%$ & $54 \%$ & $=$ & $=$ & + & + & $0 \%$ & $6 \%$ & $=$ & $=$ & $=$ & + & $0 \%$ & $1 \%$ \\
\hline Ciência da Computação & - & + & + & & $22 \%$ & $31 \%$ & $=$ & $=$ & $=$ & $=$ & $0 \%$ & $0 \%$ & $=$ & $=$ & $=$ & $=$ & $0 \%$ & $0 \%$ \\
\hline Matemática & $=$ & + & - & + & $0 \%$ & $17 \%$ & + & - & + & - & $0 \%$ & $7 \%$ & $=$ & $=$ & + & - & $0 \%$ & $0 \%$ \\
\hline Física & $=$ & $=$ & $=$ & + & $0 \%$ & $9 \%$ & + & + & + & - & $0 \%$ & $15 \%$ & - & $=$ & $=$ & $=$ & $0 \%$ & $1 \%$ \\
\hline Ciência do Espaço & $=$ & $=$ & $=$ & $=$ & $0 \%$ & $0 \%$ & $=$ & $=$ & $=$ & $=$ & $0 \%$ & $0 \%$ & $=$ & $=$ & $=$ & $=$ & $0 \%$ & $0 \%$ \\
\hline Imunologia & $=$ & $=$ & $=$ & $=$ & $0 \%$ & $0 \%$ & $=$ & $=$ & $=$ & $=$ & $0 \%$ & $0 \%$ & $=$ & $=$ & $=$ & $=$ & $0 \%$ & $0 \%$ \\
\hline
\end{tabular}

Fonte: Elaborado pelos autores (2014).

Nota: Foram utilizados sinais indicando se as mudanças entre triênios subsequentes foram de "+" (crescimento), "-" (decrescimento) ou "=" (inalterado). Valores mínimo (mín) e máximo (máx) correspondem aos valores extremos observados entre os triênios, não importando qual. 
estudo, as Ciências Sociais incluem a área de Saúde Pública, entre cujos critérios de avaliação figura um considerável Fator de Impacto de valor 4,0 para classificação de revistas A1, o que pode explicar a diminuição percentual de publicações em revistas nacionais da zona 3, nos últimos quatro triênios. O mesmo se dá na área de Humanas, na qual se identifica o critério vinculado ao Fator de Impacto mínimo de 0,5 na área de Geografia, para classificação de revistas A1 (Mugnaini \& Sales, 2011).

Analisando a Tabela como um todo, pode-se perceber que o número de aumentos (+) e diminuições (-) das diversas áreas, entre os triênios, é praticamente o mesmo, considerando-se a zona 1. Porém, ao se analisar a zona 2, os aumentos prevalecem, enquanto na zona 3, contrariamente, predominam os decréscimos. Tais constatações reforçam o efeito acima citado sobre a avaliação nacional.

Quando se observam as áreas específicas, ordenadas pelos percentuais máximo e mínimo de artigos em revistas nacionais da zona 1, nota-se as já mencionadas áreas nas quais há predomínio absoluto, assim como as áreas com maior percentual observado na zona 3. Dentre essas áreas, convém destacar as Ciências Agrárias, nas quais Fink et al. (2014) observaram um aumento de produção em relação à média mundial no período entre 2000 e 2009, o que segundo os autores não se expressou de maneira similar nas citações (apesar de serem o dobro da média mundial). Eles arriscam afirmar que talvez a ausência de aumento em citações pode se dever à especificidade da pesquisa nessa área, que pode estar direcionada a estudos de problemas locais, de menor interesse para o restante do mundo. Tal afirmação encontra respaldo neste estudo, na medida em que se observa a importância das revistas nacionais, predominando as zonas 1 e 2 .

Em seguida vêm as áreas de saúde e biológicas que, em sua maioria, mostram diminuição desse percentual na zona 1, sinalizando um predomínio de internacionalização da pesquisa nos últimos triênios. Para as áreas de Biologia e Bioquímica, Biologia Molecular e Genética e Neurociência e Comportamento esses efeitos são observados em triênios anteriores, diferente de Medicina Clínica, cujo efeito só é notado entre os últimos dois triênios. Fink et al. (2014) identificam Ambiente/Ecologia, Farmacologia e Toxicologia e Microbiologia como áreas que mantêm suas posições em relação à média mundial, fazendo-o de maneira balanceada, considerando produção e citação. Essas são áreas cujas zonas 2 e 3 são predominantemente internacionais, sendo que as duas primeiras vêm apresentando maior participação das revistas nacionais na zona 2 ao longo dos triênios.

Entre as áreas de exatas e da terra, que se agrupam na parte inferior da Tabela, destacam-se Geociências, Engenharia, Matemática e Física, por aumentos apresentados nas zonas 1 e 2, em diversos triênios. Na base da Tabela, Imunologia e Ciência do Espaço destacam-se pela publicação exclusivamente internacional.

\section{Conclusão}

Nos últimos quinze anos, a produção científica brasileira tem logrado melhores posições no cenário mundial, graças aos esforços que o país vem empreendendo em nível nacional. Os investimentos em formação e aperfeiçoamento de pesquisadores têm estimulado tanto a atividade científica em si quanto as atividades relacionadas à editoria de revistas nacionais. Os resultados deste estudo evidenciam que a produção tem avançado crescentemente às revistas estrangeiras, ao mesmo tempo que as revistas nacionais desempenham importante papel na infraestrutura de comunicação científica, tanto no âmbito nacional quanto internacional.

A complexidade associada ao processo de avaliação da produção científica se dá, em grande parte, pelas características das diversas áreas da ciência. Como se pôde perceber, o papel das revistas nacionais reforça ainda mais tais diferenças, na medida em que algumas áreas são menos internacionalizadas que outras. Há que se considerar ainda que este estudo limitou-se apenas aos artigos em periódicos, desconsiderando que algumas áreas costumam publicar mais em livros ou anais de eventos.

Outro aspecto diz respeito às zonas de Bradford que, num primeiro momento, revelaram que algumas áreas publicam num conjunto de revistas consideravelmente maior do que as demais, como Medicina Clinica. Outras áreas destacam-se pelo fato de apresentarem dispersão de sua produção em maior número de revistas da zona 3, que apresentam baixa frequência de artigos, 
evidenciando a maior variedade de revistas para publicação, como é o caso de Ciências Sociais, Psiquiatria/ Psicologia e Economia e Negócios.

Notou-se ainda um aumento mais generalizado do número de revistas das áreas, sucessivamente, na zona 3 (entre os triênios 2 e 3), zona 2 (entre os triênios 3 e 4) e zona 1 (entre os triênios 4 e 5). Associou-se a esse fenômeno o processo de avaliação Qualis, cuja lista de revistas aumenta a cada triênio, à medida em que a produção das áreas se dispersa por novos títulos, sendo esse o efeito mensurado no presente estudo. Além disso, a classificação do Qualis segundo estratos acaba distinguindo um grupo de revistas mais importantes, podendo explicar o aumento do número de revistas das zonas 1 e 2, com maior frequência de artigos.

Finalmente, a análise da variação do percentual da produção das áreas em revistas nacionais diferenciou três grupos de áreas. Na área de ciências sociais e humanidades, as zonas 1 e 2 são exclusivamente compostas por revistas nacionais, enquanto a zona 3 vem apresentando diminuição de percentual, devido ao esforço de publicação em revistas estrangeiras. Na área de Física, Ciência do Espaço e Imunologia, a produção se dá em revistas internacionais, não importando a zona. Já as demais áreas de ciências exatas e da terra, saúde e biológicas vêm apresentando aumento do percentual de publicação em revistas nacionais nas zonas 1 e 2, permanecendo a zona 3 exclusivamente internacional.

Pôde-se concluir que o fluxo da produção tem sido influenciado pelas revistas nacionais, cujo reconhecimento tem sido atestado não apenas pela inserção na Web of Science, mas pela própria comunidade científica quando da definição dos critérios de classificação de revistas no Qualis.

A metodologia utilizada neste trabalho permite a análise da produção científica nacional, utilizando Zonas de Bradford sobre um longo período de tempo. Destaca-se que, em continuação, a pesquisa aplicará a mesma metodologia para avaliação do consumo de informação das publicações nacionais, com base nas referências bibliográficas, com o objetivo de prover a política científica nacional (Qualis) de indicadores dinâmicos e contextualizados das revistas utilizadas.

\section{Agradecimentos}

À Fundação de Amparo à Pesquisa do Estado de São Paulo (Projeto Jovens Pesquisadores 2012/00255-6) e ao Conselho Nacional de Desenvolvimento Científico e Tecnológico (Projeto Universal 306046/2013-0 e Bolsa de Produtividade em Pesquisa 477246/2013-3).

\section{Referências}

Abrizah, A., et al. Citation performance of Malaysian scholarly journals in the Web of Science, 2006-2010. Serials Review, v.39, n.1, p.47-55, 2013.

Bradford, S.C. Documentação. Rio de Janeiro: Fundo de Cultura, 1961. p.196-216.

Collazo-Reyes, F. Growth of the number of indexed journals of Latin America and the Caribbean: The effect on the impact of each country. Scientometrics, v.89, n.1, p.197-209, 2013.

Coordenação de Aperfeiçoamento de Pessoal de Nível Superior. Webqualis Capes. 2014. Disponível em <http:// www.capes.gov.br/avaliacao/qualis>. Acesso em: 12 jan. 2014.

Costa, T.; Vaz, F.; Lopes, C. The impact of the online knowledge library: Its use and impact on the production of the Portuguese academic and scientific community (2000-2010). In: Qualitative and Quantitative Methods in Libraries International Conference, 5., 2013, Roma. Eletronic proceedings... Roma, 2013. Available from: <http://repositorio.ispa.pt/handle/10400.12/ 2592>. Cited: Aug. 8, 2014.
Fink, D. et al. S\&T knowledge production from 2000 to 2009 in two periphery countries: Brazil and South Korea. Scientometrics, v.99, n.1, p.37-54, 2014.

Guimarães, J.A.; Humann, M. C. Training of human-resources in science and technology in Brazil: The importance of a vigorous postgraduate program and its impact on the development of the country. Scientometrics, v.34, n.1, p.101-119, 1995.

Huh, S. Citation analysis of the Korean Journal of Urology from Web of Science, Scopus, Korean Medical Citation Index, KoreaMed Synapse, and Google Scholar. Korean Journal of Urology, v.54, n.4, p.220-228, 2013.

Internacional Society for Scientometrics and Informetrics. Past Conferences. 2014. Available from:: <http://www.issi-society. org/past.html>. Cited: Jan. 12, 2014.

Leite, P.; Mugnaime, R.; Leta, J. A new indicator for international visibility: Exploring Brazilian scientific community. Scientometrics, v.88, n.1, p.311-319, 2011. 
Leta, J. Indicadores de desempenho, ciência brasileira e a cobertura das bases informacionais. Revista USP, n.89, p.62-67, 2011.

Leta, J. Brazilian growth in the mainstream science: The role of human resources and national journals. Journal of Scientometric Research, v.1, n. 1, p.44-52, 2012.

Milanez, D. H. Nanotecnologia: indicadores tecnológicos sobre os avanços em materiais a partir da análise de documentos de patentes. 2011. Dissertação (Mestrado em Ciência e Engenharia de Materiais) - Centro de Ciências Exatas e de Tecnologia de Materiais, Universidade Federal de São Carlos, 2011.

Miranda, E.C.; Mugnaini, R. Scientific policy in Brazil: Exploratory analysis of assessment criteria. In: International Society of Scientometrics and Informetrics Conference, 14., 2013, Viena. Proceedings... Viena: Austrian Institute of Technology $\mathrm{GmbH}$, 2013. v.1. p.1578-1586.

Morel, R.; Morel, C. Um estudo sobre a produção científica brasileira, segundo os dados do Institute for Scientific Information (ISI). Ciência da Informação, v.6, n.2, p.99-109, 1977.

Mugnaini, R. 40 anos de bibliometria no Brasil: da bibliografia estatística à avaliação da produção científica nacional. In: Hayashi, M.C.P.I.; Leta, J. (Ed.). Bibliometria e cientometria: reflexões teóricas e interfaces. São Carlos: Pedro e João Editores, 2013. p.37-58.

Mugnaini, R.; Sales, D.P. Mapeamento do uso de índices de citação e indicadores bibliométricos na avaliação da produção científica brasileira. In: Encontro Nacional de Pesquisa em Ciência da Informação, 12., 2011, Brasília. Anais... Brasília: Thesaurus, 2011. v.12. p.2361-2372.

Mugnaini, R. et al. Comunicação científica no Brasil (19982012): infraestrutura nacional e internacionalização. In: Congreso Internacional de Información INFO'2014, 13., 2014, Havana. Anais... Havana: Consultoría BioMundi/IDICT, 2014. 1 CD-Rom.

Packer, A.L. Os periódicos brasileiros e a comunicação da pesquisa nacional. Revista USP, n.89, p.26-61, 2011.
Packer, A.L. SciELO citation index no Web of Science. SciELO em Perspectiva.São Paulo, 27 fev. 2014. Disponível em: <http:// blog.scielo.org/blog/2014/02/28/scielo-citation-index-noweb-of-science/>. Acesso em: 8 ago. 2014.

Packer, A.L.; Meneghini, R. Learning to communicate science in developing countries. Interciencia, v.32, n.9, p.643-647, 2007.

Pikic, A.; Mayer, M.; Macan, B. (Inter) national orientation of croatian Social Sciences and Arts and Humanities Journals Indexed in the Web of Science Database. Društvena Istraživanja-Casopis za Opca Društvena Pitanja, v.21, n.2, p.505-521, 2012.

Purnell, P.J.; Quevedo-Blasco, R. Benefits to the Spanish research community of regional content expansion in Web of Science. International Journal of Clinical and Health Psychology, v.13, n.2, p.147-154, 2013.

Quevedo-Blasco, R. Revistas iberoamericanas de psicología indexadas en el Journal Citation Reports de 2011. Revista Mexicana de Psicología, v.30, p.1-10, 2013.

Rojas-Sola, J.I.; de San-Antonio-Gomez, C. Análisis bibliométrico de las publicaciones científicas colombianas en la categoría engineering, multidisciplinary de la base de datos Web of Science (1997-2009). Dyna, v.77, n.164, p.9-17, 2010.

Souza, E.P.; Paula, M.C.S. Qualis: a base de qualificação dos periódicos científicos utilizada na avaliação Capes. Infocapes, v.10, n.2, p.7-25, 2002.

Testa, J. The globalization of Web of Science. Thomson Reuters Expert Essays. 2011. Available from: <http://wokinfo.com/ products_tools/multidisciplinary/webofscience/contentexp/ expansionessay/>. Cited: Jan. 12, 2014.

Thomson Reuters. Science Watch. 2013. Available from: <http:// archive.sciencewatch.com/about/met/>. Cited: Dez. 22, 2013.

Thomson Reuters. Web of Science. 2014. Available from: <http://images.webofknowledge.com/WOKRS513R8.1/help/ pt_BR/WOK/hs_research_tools.html>. Cited: Aug. 8, 2014.

Wolfram, D. An analysis of canadian contributions to the Information Science Research Literature: 1989-2008. Canadian Journal of Information and Library Science, v.36, n.1, p.52-66, 2012. 
